\title{
Granuloma Gravidarum at Unusual Site
}

\author{
Capt Ankit Gupta ${ }^{1}$, Col SK Rath², Capt Umesh kumar ${ }^{3}$
}

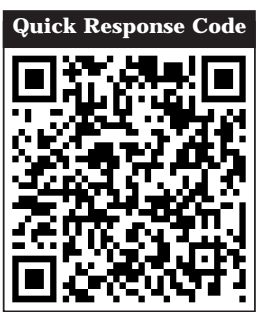

doi: $10.5866 / 2016.8 .10176$

1\&32Dental Officer

9 Corps Dental Unit, Yol Cantt

${ }^{2}$ Classified Spl (Periodontics)

Commanding Officer and Corps Dental Advisor

9 Corps Dental Unit, Yol Cantt

\section{Article Info:}

Received: J uly 9, 2016

Review Completed: August 8, 2016

Accepted: September 10, 2016

Available Online: December, 2016 (www.nacd.in)

(C) NAD, 2016 - All rights reserved

\section{Email for correspondence:}

umeshchandolia@rediffmail.com

\begin{abstract}
:
Granuloma gravidarum (GG) is an inflammatory lesion, which develops in the oral mucosa of pregnant women in response to chronic irritants of low degree such as bacterial biofilm, dental calculus and traumatic agents. Increased concentrations of estrogen and progesterone raises the levels of Prevotella intermedia in the subgingival biofilm, diminishes the host response to bacterial biofilm, increases vascular permeability, favors theinfiltration of fluids into the perivascular tissues and enhances the inflammatory response, having an important role in the development of the lesion.
\end{abstract}

Key words: Granuloma gravidarum biofilm granuloma telangiectaticum

\section{INTRODUCTION}

Granuloma gravidarum (GG) is an inflammatory lesion, which develops in the oral mucosa of pregnant women in response to chronic irritants of low degree such as bacterial biofilm, dental calculus and traumatic agents. Increased concentrations of estrogen and progesterone raises thelevels of Prevotella intermedia in the subgingival biofilm, diminishes the host response to bacterial bi ofilm, increases vascular permeability, favors the infiltration of fluids into the perivascular tissues and enhances the inflammatory response, having an important role in the development of the lesion.

Granuloma gravidarum is a benign friable neoplasm of the oral mucosa which occurs during pregnancy. It is a self limiting condition. Generally, only symptomatic treatment is required. Destructive technique is needed only if it is causing inconvenience. Hallmark of the disease is a non healing red nodule that bleeds profusely with minor trauma and does not heal. Other synonyms of granuloma gravidarum are epulis gravidarum, granuloma pyogenicum, granuloma telangiectaticum, pregnancy tumor, Iobular capillary hemangioma, pyogenic granuloma.

\section{CASE REPORT}

A twenty three year-old female reported to the Outpatient Department, complaining of a swelling in the lower right back region on inner side. On eliciting the history of present illness, the patient reported that swelling originated at 14th week of pregnancy which was painless and gradually 
increased to the present size after a normal delivery before three months. During this period she had visited a medical specialist who had advised her gum paint for application. She had stopped brushing the area due to bleeding from the area. There was no relevant past medical history which signifies that the patient was not suffering from diabetes, tuberculosis, hypertension nor the patient was allergic to any medications. Personal history reveals that the patient was brushing her teeth twice daily with conventional mechanical brushing and the baby born was her first issue. N one of her family members had this type of lesion before.

On extra oral examination, there was no visible swelling or deformity of face. Intraoral examination revealed a large pedunculated gingival overgrowth extending on lingual surface of lower right premolar and first molar region (Figure 1). Peduncle was attached on thelingual surfaces inter dental locality of 44 and 45 . It was reddish pink in color and was approximately $21 \mathrm{~mm} \times 44 \mathrm{~mm}$ in size. The surface was smooth without any ulcerations and it appeared ovoid in shape. Oral hygiene was poor and the mouth showed large amounts of calculus. No other abnormalities were observed throughout the oral mucosa with full components of dentition.

On palpation, swelling was hard, firm, movable, non pulsatile, non fluctuant and non tender in nature and the teeth associated with it did not show

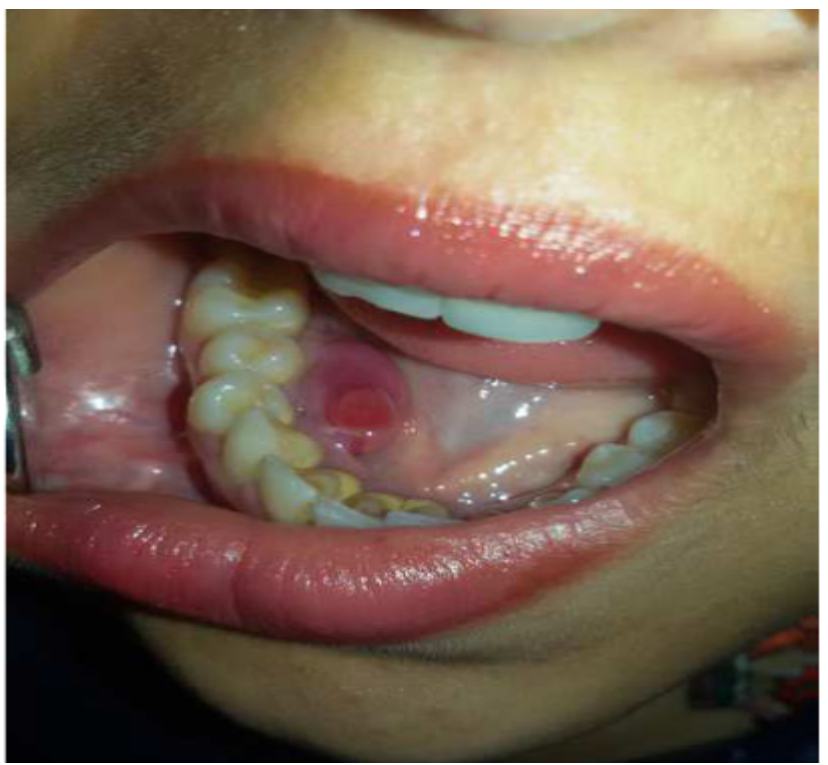

Figure 1: Clinical picture showing a large pedunculated gingival overgrowth extending on lingual surface of lower right premolar and first molar region. any mobility. No localized lymphadenopathy was elicited. Radiographically, there were no visible abnormalities and the alveolar bone in the region of the growth appeared normal. No sign of bone resorption was marked related to the site of lesion. Her general examination, systemic examination and routine hemogram comprising of TLC, DLC, $\mathrm{Hb}$ were all well within normal limits.

Based on history, clinical examination, a provisional diagnosis of Granuloma Gravidarum was made. The differential diagnosis included periapical abscess, peripheral ossifying fibroma, peripheral giant cell granuloma, localized hemangioma. Basic periodontal treatment, including scaling, polishing, and instructions on oral hygiene were carried out at the first visit. In the first phase of the treatment, ultrasonic scaling was done. Proper oral hygiene instructions were given, tooth brushing technique was demonstrated, chlorhexidine mouthwash was prescribed, warm saline rinses was advised for the patient and was recalled after 1 week.

In the surgical phase of therapy, excisional biopsy was done. The lesion was removed under the influence of $2 \%$ Lignocaine HCL with adrenaline by the use of a No 15 surgical bladein Toto. After tissue excision, residual calculus was removed and root planing was done. 3-0 black silk suture was given interdentally and the tissue was approximated.

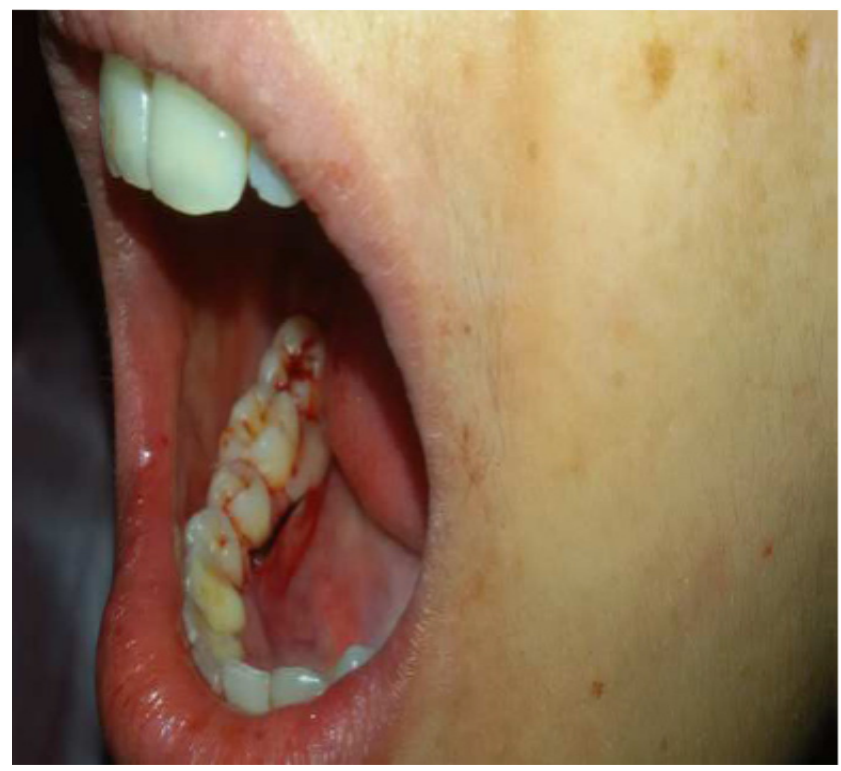

Figure 2: Clinical picture showing no signs of recurrence even after 1 year. 
Patient was given Augmentin 625 mg twice a day and Brufen 400mg as and when required. The patient was encouraged to maintain normal hygiene and to rinse her mouth with 0.2 chlorhexidine solution, twice a day for 7 days. The excised tissue was sent for histopathological examination to pathology lab.

The histopathology indicated the features of loose granulation tissue with proliferation of endothelial cells, accompanied by a fibrosis that confirmed the diagnosis to be Granuloma Gravidarum. After a gap of 7 days the patient was recalled and sutures were removed. The patient has been under observation for last 1 year without any complication or recurrence (Figure 2).

\section{Discussion}

Gingival changes in pregnancy were described as early as 1898, even before knowledge about hormonal changes was available. Granuloma gravidarum is a benign tumor which usually appears in the second and third trimester. It is misnamed capillary proliferative lesion which is neither infectious nor granulomatous. It is composed of proliferating capillaries in a loose stroma and is relatively rich in mucin. This benign hyperplastic lesion usually appears in the oral mucosa, commonest site being mucosa of the lips and gums. ${ }^{1}$ The gingiva, especially the marginal gingival is affected more than the alveolar part. Besides the gingiva it is also noticed on the lips, tongue or buccal mucosa, affecting the maxilla more than the mandible, theanterior region than the posterior with the buccal surfaces being affected more than the lingual surfaces. ${ }^{2}$ In the case presented here the lesion appeared on lingual border of right mandible which is actually a rare site for this type of Iesion as per the available literature.

Granuloma pyogenicum of pregnancy in subcutaneous and superficial tissues have al so been reported. This rapidly growing lesion is typically a painless sessile or pedunculated mass of varied diameter with size varying from 5-10 mm. It starts as a small pin head sized lesion, which grows rapidly. It is prone to spontaneous hemorrhage or hemorrhage after minor trauma. Histologically granuloma gravidarum present loose granulation tissue rich in capillary vessels and proliferation of endothelial cells, typically accompanied by a mixture of inflammatory cells. A thin epithelial layer overlies the lesion which is often ulcerated due to trauma associated with minor injury.
The exact etiology of pyogenic granuloma is unknown. Many factors have been suggested, including hormonal influences, as many of the lesions, though not all which appear during pregnancy resolve soon after delivery. Hormonal influences (specifically of progesterone) almost certainly play a role in the pathogenesis of pyogenic granuloma because these lesions commonly devel op in pregnant women or in those taking oral contraceptives. Elevated levels of estrogen during pregnancy may play a role by direct hormone action as estrogen receptor was found weakly positive in a case reported by Demir. ${ }^{3}$ Others have suggested that other pregnancy related angiogenic factors might play a role. A viral origin has also been hypothesized but seems unlikely because the most common types of human papilloma virus have been ruled out as etiol ogic agents by polymerase chain reaction (PCR) testing. Kornman \& L oesch (1980) have reported that the subgingival flora changes to a more anaerobic flora as pregnancy progresses. Prevotella intermedia is the only microorganism that increases significantly during pregnancy. They also stated that the increase is due to el evations of levels of systemic estradiol and progesterone. O'N eil (1979) suggested that the altered tissue response to plaque is due to depression of the maternal T-Iymphocyte.

Treatment considerations during pregnancy are very important as these lesions are more common duringpregnancy. Oral hygiene maintenance and regular follow up are recommended. Recurrences are believed to result from incomplete excision, failure to remove etiologic factors or injury to the area. ${ }^{4}$ Most of the pregnancy tumours regress after pregnancy. Treatment during pregnancy is only needed if the lesion causes irritation or bleeding. Therapeutic alternatives can be in the form of a destructive technique using a laser, electro-cautery, cryocautery, or chemical cautery. The present case was treated successfully with excision and has been observed forapproximately one year with good results without any signs of recurrence.

\section{References}

1. Pradhan KA, Bhoraskar N, Palnitkar A. Granuloma gravidarum (A case report). Ind J Surg 1989; 51:51-3.

2. Vilmann A, Vilmann P, Vilmann H. Pyogenic granuloma: Evaluation of oral conditions. Br J Oral Maxillofac Surg 1986; 24:37682.

3. Demir Y, Demir S, Aktepe F. Cutaneous Iobular capillary hemangioma induced by pregnancy. J Cutan Pathol 2004; 31:77-80.

4. Neville BW, Damm DD, Allen CM, Bouquot J E (2002). Oral and maxillofacial Pathology 2nd Ed., WB Saunders, Philadel phia, pp. 437-95. 